\title{
THE TIME-COURSE OF THE INDUCTION OF REPARATIVE DENTINE FORMATION IN MONKEYS BY RECOMBINANT HUMAN OSTEOGENIC PROTEIN-1
}

\author{
R. Bruce Rutherford, ${ }^{1 *}$ Larz Spångberg, ${ }^{2}$ Marjorie Tucker, ${ }^{3}$ David Rueger ${ }^{3}$ \\ and MARC ChaRETTE ${ }^{3}$ \\ 'University of Michigan School of Dentistry, Ann Arbor, MI 48109, ${ }^{2}$ University of Connecticut School \\ of Dental Medicine, Farmington, CT 06032 and ${ }^{3}$ Creative BioMolecules, Inc., Hopkinton, \\ MA 01748, U.S.A.
}

(Accepted 2 June 1994)

\begin{abstract}
Summary-Recombinant human osteogenic protein-1 (hOP-1, BMP-7) induces cartilage and bone formation when implanted in extra- and intraskeletal sites in vivo. rOP-1 also preserves pulp vitality and stimulates reparative dentine formation when placed on partially amputated vital dental pulp tissue. The amount of dentine formed in 6 weeks was earlier found to be proportional to the total amount of rOP-1/carrier placed on the pulp and the capacity of the pulp to respond to rOP-1 appeared to be independent of the amount of coronal pulp removed. This reparative dentine was not completely mineralized after 6 weeks healing. Experiments were now made to determine the capacity of hOP-1 to preserve the vitality of and induce reparative dentine in vital radicular pulps. The extent of tissue mineralization present after 1, 2, 4 and 6 months' healing time in permanent monkey teeth was assessed. Radicular pulp vitality was maintained, reparative dentine formed, and mineralization was nearly $75 \%$ complete after 1 month and more than $95 \%$ after 4 months. The effects of irrigating the exposed pulps with EDTA, sodium hypochlorite or saline were also compared. Significantly more sodium hypochloritetreated pulps became non-vital and the root canals of all the non-vital teeth contained bacteria at the time of sacrifice.
\end{abstract}

Key words: osteogenic protein-1, reparative dentine, vital pulp therapy.

\section{INTRODUCTION}

Osteogenic (OP) or bone morphogenetic (BMP) proteins comprise a subgroup of a larger family of structurally related proteins known as the transforming growth factor- $\beta$ superfamily. Proteins of this family are implicated in diverse biological activities involving differentiation, tissue morphogenesis, regeneration and repair. Most of the seven members of the OP or BMP subgroup (BMP-2, $-3,-4,-5,-6$, OP-1 and -2 ) were originally identified by their capacity to induce bone formation in intra- or extraskeletal sites in vivo (Sampath et al., 1990; Wang et al., 1990). At least three of these proteins also induce reparative dentine formation. Crude extracts from dog bone with BMP activity (Nakashima, 1990), recombinant human BMP-2 and -4 (M. Nakashima, personal communication) and OP-1 (Rutherford et al., 1993) stimulate the formation of a form of reparative dentine when placed directly against partially amputated vital dental pulp. Other findings implicate OP-1, BMP-3 (S. Vukocevik, personal communication), BMP-2 and -4 in tooth development (Lyons, Pelton and Hogan, 1990; Vaino et al., 1993).

*Author for correspondence.

Abbreviations: BMP, bone morphogenetic protein; $(\mathrm{h}-),(\mathrm{r}-)$, $\mathrm{OP}$, (human) (recombinant) osteogenic protein.
We earlier demonstrated the capacity of recombinant human OP-1 (hOP-1) when combined with a collagen carrier matrix to preserve pulp vitality and initiate reparative dentine formation when placed directly upon large ( $>2 \mathrm{~mm}$ dia) pulp exposures in monkeys (Rutherford et al., 1993). In those experiments, variable amounts of the coronal pulp were removed, partly replaced with different amounts of the rOP-1/collagen carrier matrix and allowed to heal for 6 weeks. The pulps remained vital and reparative dentine formed in all hOP-1/collagen carrier matrix but no carrier control-treated teeth. The reparative dentine replaced and formed in the space occupied by the hOP-1/collagen carrier matrix. Hence the amount of tissue formed was proportional to the volume or mass of the hOP-1/collagen carrier matrix placed in the tooth. This reparative dentine was incompletely mineralized by 6 weeks yet the extent of mineralization appeared to be independent of the mass of hOP-1/collagen carrier matrix placed in the tooth.

These findings showed that hOP-1/collagen carrier matrix could induce a controlled amount of reparative dentine formation regardless of the extent of coronal pulp amputation. However, it seemed possible that the adult radicular pulp might not respond to hOP-1 in the same manner as the coronal pulp, as pulpotomies (full amputation of the coronal pulp) followed by calcium hydroxide induction of 
reparative dentine at the root canal orifice have a poor prognosis and therefore are contraindicated in adult human teeth (Seltzer and Bender, 1984). Therefore, we have now tested the capacity of hOP-1 to preserve pulp vitality and induce reparative dentine formation when placed directly against the surface of freshly amputated radicular pulps after complete coronal pulpectomy. The effects of treating the residual pulps with solutions of EDTA followed by sodium hypochlorite or saline were also determined. EDTA was used to remove the smear layer formed on the cut dentine surface. Sodium hypochlorite was tested because, as a disinfectant, it may be used in cases of carious or near carious exposures so we determined its effects on non-infected pulps. The number of vital teeth and the extent of mineralization after 1, 2, 4 and 6 months' healing was measured histomorphometrically.

\section{MATERIALS AND METHODS}

The production and purification of hOP-1 and the bovine bone type $I$ collagen carrier matrix, as well as the formulation of the hOP-1/collagen carrier matrix combination $(2.5 \mu \mathrm{g} \mathrm{rOP}-1 / \mathrm{mg}$ collagen $)$, were as described by Rutherford et al. (1993).

All procedures involving animals were approved by the University of Connecticut Animal Care Committee and done in an accredited animal-care facility with extensive experience in managing non-human primates. The direct pulp-capping experiments used four adolescent (mixed dentition) male Macaca fasic ularis of approx. $6-8 \mathrm{~kg}$ each. All procedures were done on animals heavily sedated with ketamine $(15 \mathrm{mg} / \mathrm{kg}$ body wt) and acepromazine $(0.55 \mathrm{mg} / \mathrm{kg}$ body wt) supplemented with intraoral infiltration anaesthesia.

Sixty-four incisor, premolar and molar teeth in four animals were isolated by rubber dam, cleaned with a $5 \%$ solution of iodine and the pulps exposed using sterile high-speed rotary cutting instruments with water spray coolant. The coronal pulps were amputated using sterile steel burs in slow rotary cutting instruments to the level of the orifice of the root canal(s). The orifice of each canal was enlarged with a No. 2 round bur to a depth of approx. 1-2 mm. Half the pulp chambers were exposed to either a sterile $17 \%$ solution of EDTA (pH 7.4) or normal saline, followed by rinsing either with a $1.0 \%$ solution of sodium hypochlorite or saline. Excess moisture was removed with sterile paper points. The pulps were then covered with 1 to $2 \mathrm{mg}$ of $\mathrm{rOP}-1 / \mathrm{mg}$ collagen carrier matrix $(2.5 \mu \mathrm{g}$ hOP-1/collagen carrier matrix, Creative BioMolecules, Hopkinton, MA) or collagen carrier matrix without hOP-1 premoistened with sterile saline and carried to the tooth with a Messing gun. The pulp chambers were then filled with Ketac Silver (ESPE-Premier, Norristown, PA) and the cavities sealed with dental amalgam (Kerr U.S.A., Romulus, MI). The animals were fed laboratory chow, observed daily for signs of abnormal behaviour and examined twice monthly under sedation. No changes in behaviour by any of the animals were noted during healing. Food intake remained normal and body weights increased in a manner consistent with normal growth. Fifty hOP-1/collagen carrier matrix- and eight collagen carrier matrix-treated teeth were analysed histologically for reparative dentine formation while dentine from the remaining teeth was processed for biochemical analyses (data not shown). The collagen-based carrier was inactive in all previous investigations (Rutherford et al., 1993). Therefore only enough collagen carrier matrixtreated teeth to provide two controls at each time interval were included, in order to conserve animals. Each animal received two sets of treatment conditions staged so that half the teeth from two animals healed for 1 or 2 months and half the teeth from the other two animals healed for 4 or 6 months (Table 1).

Three animals were anaesthetized at the specified times after surgery and perfused with phosphate-

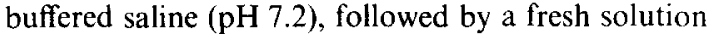
of $4 \%$ paraformaldehyde $(4 \mathrm{C})$ in phosphatebuffered saline ( $\mathrm{pH} 7.2$ ). The jaws were resected and the samples prepared by removing each experimental tooth in block. The samples were further fixed by immersion in fresh $4 \%$ paraformaldehyde in phosphate-buffered saline ( $\mathrm{pH} 7.2$ ) and demineralized in formic acid/sodium citrate at room temperature to a radiological end-point. Block specimens containing six teeth (four maxillary incisors and two maxillary premolars, with normal periapical radiographic ana1omy and radiographic evidence of reparative dentine formation) were removed from the anaesthetized fourth animal before fixation, quick frozen in liquid nitrogen and stored at $-80^{\circ} \mathrm{C}$ for the biochemical assays (data not reported). The fixed specimens were processed, embedded in parattin, serially sectioned (5-7 $\mu \mathrm{m})$ and some sections were stained with haematoxylin and eosin and others for bacteria (Preston and Morrell, 1962).

Computer-assisted histomorphometric analysis was made by capturing video images (video camera, Model 65, Dage-MTI, Michigan City, IN) of the histological preparations taken through the microscope. Quick Capture (Data Translation, Inc., Marlboro, MA) installed in a Macintosh II (Apple Computer, Cuppertino, CA) connected to the video

Table 1. Distribution of teeth by treatment groups and healing times

\begin{tabular}{lccccc}
\hline Animal & OP-I; CM $^{*}$ & $\begin{array}{c}\text { Healing } \\
\text { period }\end{array}$ & OP-1; $\mathrm{CM}^{*}$ & $\begin{array}{c}\text { Healing } \\
\text { period }\end{array}$ & $\begin{array}{c}\text { Teeth } \\
\text { collected }\end{array}$ \\
\hline 1 & $7 ; 1$ & 2 mos & $7: 1$ & 4 mos & All \\
2 & $7 ; 1$ & 2 mos & $7: 1$ & 4 mos & All \\
3 & $7 ; 1$ & 1 mo & $7 ; 1$ & 1 mo & All \\
4 & $7 ; 1$ & 1 mo & $7 ; 1$ & 1 mo & All \\
\hline
\end{tabular}

$\mathrm{CM}$, collagen carrier matrix; OP-1. osteogenic protein-1.

*Values indicate the number of teeth treated with rOP-1/CM (OP-1) and teeth treated with carrier matrix without rOP-1 (CM). 
camera was used to digitize the images. These images were analysed using Image 1.4 software calibrated with a stage micrometer. Specific areas of the tissues, e.g. those mineralized and those not mineralized as well as the junction between the tubular dentine and the mineralized reparative dentine, which are clearly displayed on high resolution computer video screens, were electronically outlined and the areas determined by program algorithms. Five to eight sections representing more than $75 \%$ of the mass of reparative dentine were analysed. In all sections with vital pulp, the reparative dentine extended to the dentine walls surrounding the prepared cavity.

\section{RESULTS}

Seven rOP-1/collagen carrier matrix-treated teeth lost vitality during this study in contrast to our previous experiments in which all pulps treated with rOP-1/collagen carrier matrix remained vital and formed reparative dentine (Rutherford et al., 1993). In contrast, reparative dentine failed to form in all collagen carrier matrix-treated teeth, half of which (4 and 6 months) were non-vital at death. These tissues appeared identical to collagen carrier matrix-treated pulps described by Rutherford et al. (1993). Irrigating the pulps with EDTA had no measurable effect on pulp vitality or reparative dentine formation (Table 2) while significantly more of the sodium hypochlorite-treated group became non-vital (Table 3 ). No obvious patterns emerged from the evaluation of the non-vital teeth. Equal numbers of incisors and molars were treated and three incisors and four molars became non-vital. All the non-vital teeth except one were detected histologically, preventing any estimate of a distribution of these non-vital teeth according to duration of treatment. In three of the seven non-vital teeth, small amounts of reparative dentine formed but did not extend to all margins of the prepared sites. A single periapical abscess was detected clinically at 3 weeks and the tooth extracted. The root canals of all non-vital teeth but no vital teeth stained positively for bacteria (data not shown). In all cases, treated teeth immediately adjacent to those that became non-vital remained vital and formed reparative dentine.

The extent of mineralization was measured histomorphologically after each healing interval (Fig. 1). Mineralized tissue was judged from the staining characteristics and the absence of definable soft tissue elements. Lacunae in which single cells or vessels were completely surrounded by mineralized tissue were included in the mineralized category. In contrast, lacunae containing non-mineralized extracellular matrix with or without cell profiles and/or vessels were categorized as non-mineralized (Figs 2 and 3). The differences in the extent of mineralization of

Table 2. Effect of irrigation solutions on vitality of rOP-1 treated teeth

\begin{tabular}{llrc}
\hline & & Vital & Non-vital \\
\hline EDTA & Na hypochlorite & 9 & 3 \\
& Saline & 12 & 1 \\
Saline & Na hypochlorite & 9 & 3 \\
& Saline & 13 & 0 \\
\hline
\end{tabular}

Table 3. Percentage of viable pulps after treatment with different irrigants

\begin{tabular}{lc}
\hline Treatment & $\begin{array}{c}\text { Viable pulps } \\
\text { with reparative dentine* }\end{array}$ \\
\hline Saline & $96 \% \%^{* *}$ \\
Sodium hypochlorite & $75 \%$ \\
\hline
\end{tabular}

${ }^{*} N$ equals 26 for saline and 24 for sodium hypochloritetreated teeth.

**Statistically different from sodium hypochlorite-treated teeth ( $p=0.04$, Fisher's exact test).

the tissue at 1 and 4 or 6 months are readily evident (Figs 2 and 3 ).

The effects of treating the pulps with EDTA were tested because it might be beneficial to remove the dentine smear layer produced while preparing the site to receive the treatment material. However, no obvious morphological distinction was discernible at the junction of the two tissues in EDTA- or saline-treated teeth. Separation of the two tissues is evident in some sections from both the EDTA- and saline-treated groups but no significant differences were evident (Figs 2 and 3). We did not attempt to measure the adherence of the reparative dentine to the prepared secondary dentine.

Two distinct morphological types of reparative dentine could be identified in most sections after 2 months' healing. One type appeared to be without identifiable tubules and to be associated with flattened cells at the mineralized front (Fig. 5). We refer to this tissue as atubular-like dentine. The other type appeared as small foci of tubular-like dentine associated with more columnar cells at the mineralizing front (Fig. 4). This tubular-like reparative dentine never appeared to occupy more than $10-20 \%$ of the cross-sectional area of any histological section. Zones of residual secondary dentine powder produced during cavity preparation remained after irrigation and were incorporated into the mineralized matrix of the reparative dentine (Figs 3 and 4).

In our previous study (Rutherford et al., 1993), the treated teeth were filled to the cavosurface with

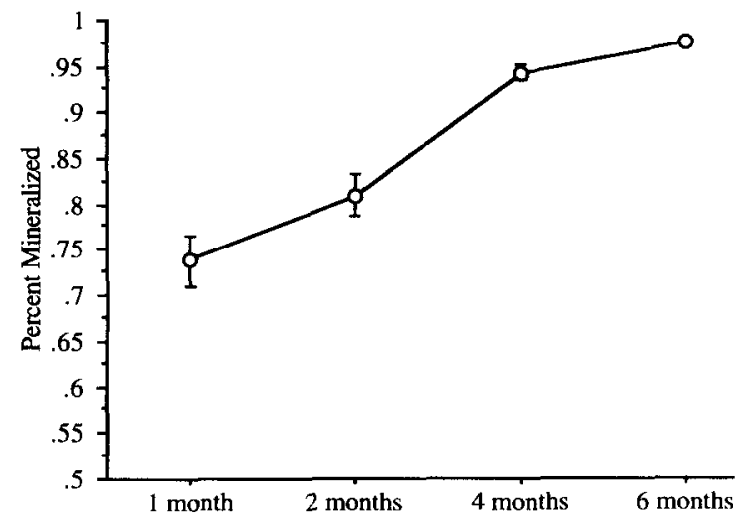

Fig. 1. Values are mean percent $\pm S E$ of the portion of the tissue mineralized in 5-8 cross-sections spanning greater than $75 \%$ of the reparative dentine from each of three teeth selected randomly from each healing interval. The values at 4 and 6 months are statistically different from those at 1 and 2 months $(p<0.001)$ but not from each other. 

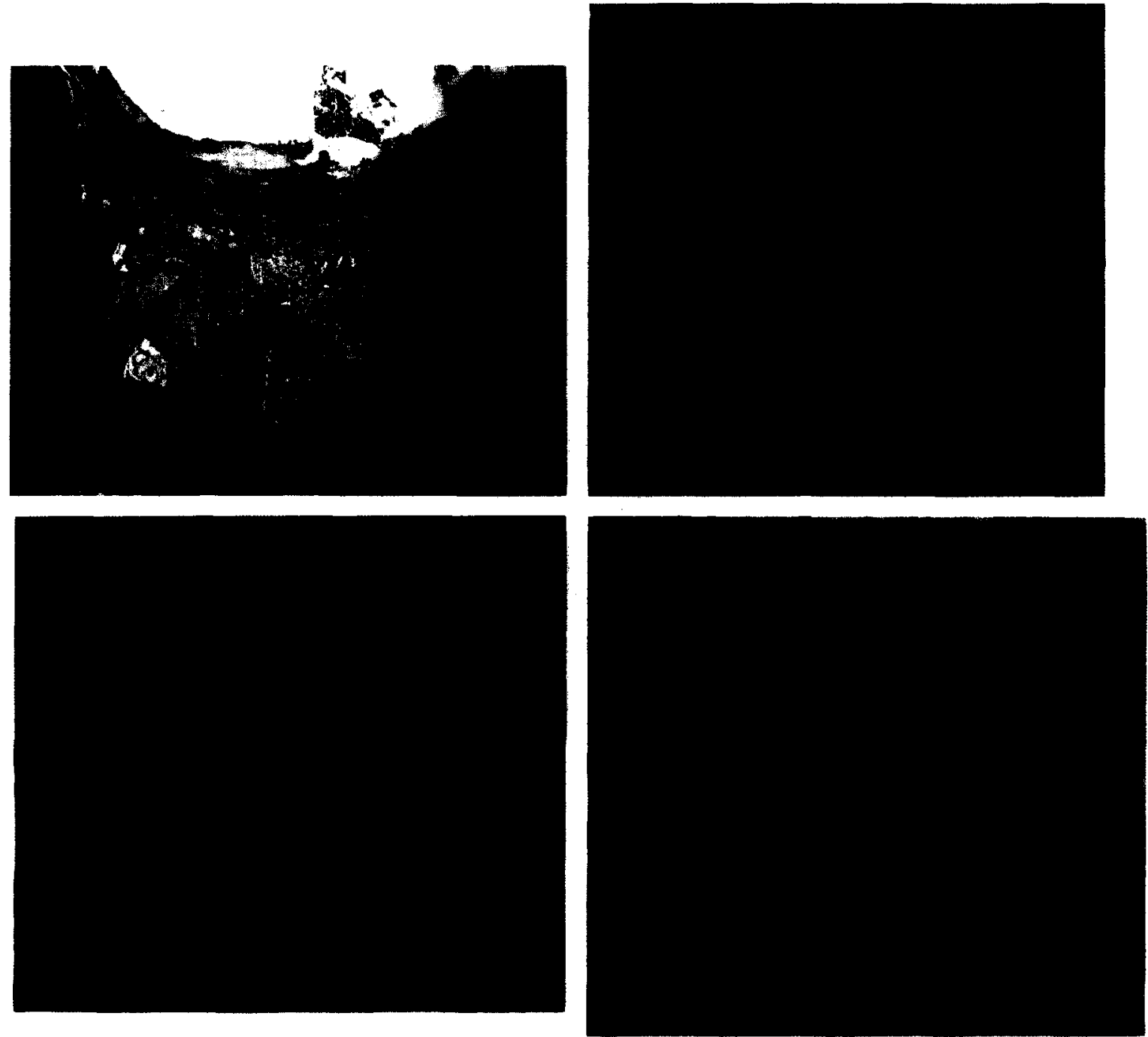

Fig. 2. Photomicrograph of a hOP-1/collagen carrier matrix-treated lesion after I month of healing. Zones of mineralized matrix $(\mathrm{M})$ are clearly evident interspersed with zones of unmineralized soft pulp tissue (ST). Such zones were not categorized as mineralized in the histomorphometric determination of the percent of tissue mineralized (Fig. 1). The prepared margin of the cavity is clearly evident (arrow). This specimen was not treated with EDTA. Bar equals $500 \mu \mathrm{m}$. Original magnification $12.5 \times$

Fig. 3. Photomicrograph of a hOP-1/collagen carrier matrix-treated lesion after 6 months' healing. In contrast to more immature tissue (Fig. 2), the mineralized matrix is interspersed with vessels and single cells which were categorized as mineralized in the histomorphometric determination of the percent of tissue mineralized (Fig. 1). The prepared margin of the cavity (arrow), the glass ionomer base, and residual dentine powder (P) are clearly evident. This specimen was treated with EDTA. Bar equals $500 \mu \mathrm{m}$. Original magnification $25 x$

Fig. 4. Photomicrograph of a hOP-1/collagen carrier matrix-treated lesion after 2 months' healing. A zone of tubular-like dentine lined by columnar odontoblast-like cells $(O)$ is evident. The separation of the odontoblast layer from the pulp is presumed to be due to preparation artifact. Bar equals $100 \mu \mathrm{m}$. Original magnification $100 \times$

Fig. 5. Photomicrograph of a hOP-1/collagen carrier matrix-treated lesion after 6 months' healing. The reparative dentine is shown separated from the wall of the root canal by a narrow band of radicular pulp tissue. The reparative dentine is lined by a layer of flattened cells without an apparent zone of predentine. In contrast the columnar odontoblasts $(O)$ lining the adjacent wall of the root canal are typically associated with a layer of predentine. Bar equals $500 \mu \mathrm{m}$. Original magnification $62.5 \times$

TempBond NE (Kerr Manufacturing, Romulus, MI), a hard-setting zinc-oxide preparation that does not contain eugenol. In all cases, reparative dentine that formed in direct contact with the TempBond appeared to be intact. In this study, the rOP1/collagen carrier matrix was covered to a depth of several millimetres with a glass ionomer cement, with no zinc oxide separating the glass ionomer from the rOP-1/collagen carrier matrix. The reparative dentine immediately subjacent to the glass ionomer base often appeared fragmented (Fig. 3). It is not clear whether this represents alterations in the reparative 
dentine forming in contact with the glass ionomer or sectioning artefact. The glass ionomer was nol completely removed from the teeth before sectioning, to avoid the possibility of mechanical damage to the specimen.

\section{DISCUSSION}

The terminology applied to tertiary dentine matrices forming in fully erupted adult teeth is confusing. Such matrices have been referred to as reactionary, reparative or, in some cases, osteodentine. We prefer to refer to the tissue formed in response to OP-1 as reparative dentine as defined by Lesot et al. (1993) and limit use of the term osteodentine to a form of dentine found in lower vertebrates (Bradford, 1967; Orvig, 1967). However, OP-1 induces alveolar bone formation when applied to fresh tooth sockets (Rutherford et al., 1992) as well as extra- and intraskeletal endochondral bone formation (Sampath et al., 1992), so we have begun a morphological and biochemical characterization of the tissue formed in dental pulps in response to hOP-1. This may permit a more definitive classification of this tissue.

After 1 month the hOP-1/collagen carrier matrixinduced reparative dentine is characterized by vascular channels and cells surrounded by an amorphous mineralized extracellular matrix. After 2 months, foci of tissue resembling tubular dentine are evident (Fig. 4). These areas, adjacent to the residual pulp, are associated with polarized cells resembling odontoblasts (Fig. 4). Tubular-like dentine was never observed forming in the absence of foci of the amorphous mineralized matrix and at 6 months no sections were observed in which this tubular-like form of dentine covered more than $10-20 \%$ of the pulpal surface. By 6 months, the reparative dentine most typically comprised large zones of atubular-like dentine lined by flattened cells (Figs 3 and 5) interspersed with zones of the tubular-like dentine. In some areas an apparent predentine zone is associated with tubular-like dentine while such a zone is not apparent adjacent to the atubular-like reparative dentine. These observations suggest that very little new tissue is forming at the pulpal surface of the reparative dentine 6 months after placing the hOP-1/collagen carrier matrix.

The factors regulating the apparent formation of differing morphological types of dentine are not clear. By light microscopy, the unresorbed bone-derived type I collagen carrier granules appear to resemble demineralized bone (not shown) as does the relatively immature reparative dentine (Fig. 3 ). It is possible that the physical structure of the carrier material could affect the morphology of the tissue formed. Therefore, it is conceivable that a collagen carrier matrix prepared by a similar process from secondary (tubular) dentine would retain some morphological features of tubular dentine and when combined with OP-1 or other dentinogenic protein induce the formation of tubular dentine. Such an effect would suggest a more active role for the carrier in tissue regeneration than is now generally envisaged.

The ability to induce selectively the formation of atubular and tubular dentine could provide models for comparing the mechanisms regulating the regen- eration of different morphological types of dentine. This capacily might also have therapeutic value, as in some clinical situations regeneration with one type of dentine or the other may be advantageous. For example, atubular dentine may be: (i) more amenable to the adherence of dentine-bonding agents, (ii) more caries resistant and (iii) less permeable than tubular dentine. In contrast, dentine tubules may be involved in preserving important biomechanical properties of dentine. Experiments testing these ideas are in progress.

Approximately $75 \%$ of the reparative tissue formed in response to rOP-1 mineralized within 1 month of treatment and was nearly completely mineralized by 4 months. Isolated lacunae containing blood vessels and single cells are readily apparent irregularly distributed throughout the reparative dentine after 6 months (Fig. 4). It remains to be determined if these tissue elements are maintained or are, over time, replaced with mineralized extracellular matrix. It is possible that some degree of cellularity and/or vascularity is important to maintaining some long-term biomechanical properties of this tissue.

Seven of 56 hOP-1/collagen carrier matrix-treated teeth became non-vital during the course of this study (Table 2). This result contrasts to our previous study where all hOP-1-treated teeth remained vital and formed reparative dentine (Rutherford et al., 1993). Irrigating the pulp with a solution of EDTA did not appear to affect qualitatively or quantitatively the pulpal response to hOP-1/collagen carrier matrix. There was no difference in the number of non-vital teeth in either the EDTA- or saline control-treated teeth. Similarly, no difference was apparent in the degree to which the new tissue was bonded to the cut surface of the secondary dentine. Separation of these tissues was observed in both groups with equal frequency and appeared more related to the amount of glass ionomer base remaining in the teeth before sectioning. It is likely that pretreatment of the cavity with EDTA is not necessary before treating pulps with hOP-1/collagen carrier matrix.

Interestingly, most of the non-vital teeth had been irrigated with sodium hypochlorite before placement of the hOP-1/collagen carrier matrix (Table 1). In our previous study the amputated coronal pulps were rinsed only with the handpiece water spray before placing the rOP-1 and the cavities in the teeth had been filled with a eugenol-free zinc oxide preparation placed directly on the rOP-1/collagen carrier matrix. In the current study the teeth were restored with a glass ionomer cement placed directly on the rOP$1 /$ collagen carrier matrix, followed by dental amalgam. Bacteria were detected in the root canals of all the non-vital teeth. This observation confounds interpretation of the utility of sodium hypochlorite as an irrigant in OP-1 vital pulp therapy because pulpal death could be due to coincidental and/or subsequent infection of the radicular pulp. However, in at least some cases, our antiseptic and/or the restorative procedures were not sufficient to prevent microbial contamination. Reparative dentine had begun to form in some of the non-vital teeth, but it is not possible to estimate the timing of the cessation of tissue formation from these data. Not surprisingly, these data reveal that some level of bacterial infection 
of the pulp is incompatible with successful formation of reparative dentine. However, the ability to induce reparative dentine formation in the radicular pulp following amputation of the coronal pulp suggests a surgical approach to treating inflamed coronal pulps. Presumably teeth with some extent of pre-existing inflammatory changes in the coronal pulp could be successfully restored by removal of the inflamed coronal pulp, formation of a limited amount of reparative dentine at the orifice of the root canal and maintenance of vital radicular pulps. Clearly the extent of pre-existing pulpal inflammation compatible with hOP-1 induction of reparative dentine formation needs to be determined. Experiments studying these questions are in progress.

\section{REFERENCES}

Bradford E. W. (1967) Structural and Chemical Organization of Teeth (Ed. Miles A. E. W.), Chap. 12, p. 3. Academic Press, London.

Lesot H., Bègue-Kirn C., Kubler M. D., Meyer J. M., Smith A. J., Cassidy N. and Ruch J. V. (1993) Experimental induction of odontoblast differentiation and stimulation during reparative processes. Cells Mal. 3, 201217.

Lyons K. M., Pelton R. W. and Hogan B. L. M. (1990) Organogenesis and pattern formation in the mouse: RNA distribution patterns suggests a role for bone morphogenetic protein-2A (BMP-2A). Development 109, 833844.

Nakashima M. (1990) lhe induction of reparative dentine in the amputated dental pulp of the dog by bone morphogenetic protein. Archs oral Biol. 35, 493497.

Orvig T. (1967) Structurat and Chemical Organization of Teeth (Ed. Miles A. E. W.). Chap. 2, p. 45. Academic Press. London.
Preston N. W. and Morrell A. (1962) Reproducible results with the gram stain. J. path. Bact. 84, 24l-243.

Rutherford R. B., Sampath T. K., Rueger D. C. and Taylor T. D. (1992) Use of bovine osteogenic protein to promote rapid osseointegration of endosseous dental implants. Int. J. oral Maxillofac. Imp. 7, 297--301.

Rutherford R. B., Wahle J., Tucker M., Rueger D. and Charette M. (1993) Induction of reparative dentine formation in monkeys by recombinant human osteogenic protein-1. Archs oral Biol. 38, 571-576.

Sampath T. K., Coughlin J. E., Whetstone R. M., Banach D. Corbett C.. Ridge R. J., Oskaynak E., Oppermann H. and Rueger D. C. (1990) Bovine osteogenic protein is composed of dimers of OP-I and BMP-2A. Two members of the transforming growth factor-B superfamily. $J$. hiol. Chem. 265, 13,198 13,205.

Sampath T. K., Maliakal J. C., Hauschka P. V., Jones W. K.. Sasak H., Tucker R. F., White K. H., Coughlin J. E., Tucker M. M. and Pang R. H. (1992) Recombinant human osteogenic protein-1 (hOP-1) induces new bone formation in vivo with a specific activity comparable with natural bovine osteogenic protein and stimulates osteoblast proliferation and differentiation in vitro. J. hiol. Chem. 267, 20.352-20.362.

Seltzer S. and Bender I. B. (1984) The Dental Pulp. J. B. Lippincott, Philadelphia.

Vaino S., Karavanova I.. Jowett A. and Thesleff I. (1993) Identification of BMP-4 as a signal mediating secondary induction between epithelial and mesenchymal tissues during early tooth development. Cell 75, 4558.

Wang E. A., Rosen V., D'Alessandro J. S., Bauduy M. Cordes P.. Harada T., Israel D. I., Hewick R. M., Kerns K. M.. LaPan P., Luxemberg G. P., McQuaid D., Moutsatsos I. K., Nove J. and Wozney J. M. (1990) Recombinant human bone morphogenetic protein induces bone formation. Proc. natn. Acad. Sci. U.S.A. 87, 2220-2224. 